\title{
A New Approach in Fractals Models
}

\author{
Deepak Negi \\ Faculty of Computer Science \\ Gyani Inder Singh Institute \\ Dehradun
}

\author{
Ashish Negi \\ Associate Professor Computer \\ Science \& Engg. Department \\ G.B.Pant Engg.College.Pauri \\ Garhwal (India).
}

\author{
Priti Dimri \\ Associate Professor Computer \\ Science \& Engg. Department \\ G.B.Pant Engg.College.Pauri \\ Garhwal (India).
}

\begin{abstract}
The term fractal was coined in 1975 by Benoit Mandelbrot, from the Latin fractus, meaning "broken" or "fractured". In colloquial usage, a fractal is a shape that is recursively constructed or self-similar, that is, a shape that appears similar at all scales of magnification and is therefore often referred to as "infinitely complex". Researchers used feedback systems to implement of a new iterative approach in the study of fractal models. The purpose of this paper is to present a review of literature in fractal analysis in recent years. In this review paper we have studied the work of various researchers in recent years on fractals models
\end{abstract}

\section{Keywords}

Complex Dynamics, Relative Superior Julia Set, Ishikawa Iteration, Relative Superior Mandelbrot Set, Relative Superior Tricorn and Relative Superior Multicorns.

\section{INTRODUCTION}

Fractals are one of the more recent discoveries in the field of mathematics. Uncovered in the late 1960s by Benoit Mandelbrot, fractals, simply put, are geometric figures that are made up of patterns and repeat themselves at smaller scales infinitely. Benoit Mandelbrot contributed greatly to the study of fractals. Prior to his work, investigations into fractals began as early as the late 1800 s, with German mathematicians George Cantor [14, 13]. Mandelbrot and other mathematicians showed through their mathematics and computer programming that fractals are also common in everyday life, in nature, our bodies, and even in popular culture, fractals are everywhere in our environments from clouds to coastlines snowflakes to seashells everywhere. Fractals are generated by an iterative process it means doing the same thing again and again. This is called self-similarity or feedback systems. Closely the whole fractal theory was developed using feedback systems.

Formally, the fractals are categorized in two types i.e. regular (geometric) and random fractals. Regular fractals consist of large and small structures that are exact copies of each other, except in size [14]. One of the more well known regular fractals is the Koch snowflake, which is made up of small triangles added to the sides of larger triangles to an infinite degree [14]. Random fractals are more apparent in nature as their small scale structures may differ in detail. It was this type of pattern that greatly influenced Mandelbrot, who gave these patterns the name fractal, from the Latin word fractus, which means a broken stone with an irregular surface. In the late 1970s, Mandelbrot began to study an equation that later became known as the Mandelbrot Set, which under computer magnification, reveals an endless succession of repeating patterns. There are two important properties of fractals are self-similarity and dimension. There are typically four techniques to generate fractals: escape-time fractals (also known as "orbits" fractals), iterated function systems, random fractals, and strange attractors [13].

\section{PRELIMINEARIES}

\subsection{Feed Back/ Iterative Procedures}

Feedback processes are fundamental in all exact sciences. In fact, they were first introduced by Sir Isaac Newton and Gottfried W. Leibniz some 300 years ago in the form of dynamic laws; and it is now standard procedure to model natural phenomena using such laws. Gaston Julia studied the iteration of polynomials and rational functions in the early twentieth century. If $f(x)$ is a function, various behaviors can arise when $f$ is iterated, we will iterate this function when initially applied to an initial value of $x$, say $x=a_{0}$. Let $a_{1}$ denote the first iterate $f\left(a_{0}\right)$, let $a_{2}$ denote the second iterate $f\left(a_{1}\right)$, which equals $f\left(f\left(a_{0}\right)\right)$, and so forth. Then we'll consider the infinite sequence of iterates $a_{0}, a_{1}=f\left(a_{0}\right), a_{2}=f\left(a_{1}\right), a_{3}=f\left(a_{2}\right)$,

There are basically two types of feedback machines, one-step machines and two-step machines. Both types of machines can be characterized by iterative procedures Iteration methods are one way to achieve the self-similarity exhibited by fractals [14]. One-step feedback machines are characterized by Peano-Picard iterations also called function iterations represented by the formula $x_{n+1}=f\left(x_{n}\right)$, where $f$ can be any function of $x$, It requires one number as input and returns a new number very useful mathematical tools and have been developed in particular for the numerical solution of complex problems. And In two-step feedback machines, the output is computed by the formula $x_{n+1}=g\left(x_{n}, x_{n-1}\right)$, which requires two numbers as input and returns a new number [13].

\section{Definition 2.1 Ishikawa Iteration [8]}

Let $X$ be a subset of real or complex numbers and $f: X \rightarrow X$ for $x_{0} \in X$, we have the sequences $\left\{x_{n}\right\}$ and $\left\{y_{n}\right\}$ in $X$ in the following manner:

$$
\begin{aligned}
& y_{n}=s_{n}^{\prime} f\left(x_{n}\right)+\left(1-s_{n}^{\prime}\right) x_{n} \\
& x_{n+1}=s_{n} f\left(y_{n}\right)+\left(1-s_{n}^{\prime}\right) x_{n}
\end{aligned}
$$


where $0 \leq s_{n}^{\prime} \leq 1,0 \leq s_{n} \leq 1$ and $s_{n}^{\prime} \& s_{n}$ are both convergent to non zero number.

\section{Definition 2.3 [15]}

The sequences $x_{n}$ and $y_{n}$ constructed above is called Ishikawa sequences of

Iterations or Relative Superior sequences of iterate. We denote it by $R S O\left(x_{0}, s_{n}, s_{n}^{\prime}, t\right)$.

Notice that $\operatorname{RSO}\left(x_{0}, s_{n}, s_{n}^{\prime}, t\right)$ with $s_{n}^{\prime}=1$ is $S O\left(x_{0}, s_{n}, t\right)$ i.e. Mann's orbit [7] and if we place:

$s_{n}=s_{n}^{\prime}=1 \quad$ Then $\quad R S O\left(x_{0}, s_{n}, s_{n}^{\prime}, t\right) \quad$ reduces to $O\left(x_{0}, t\right)$.

We remark that Ishikawa orbit $R S O\left(x_{0}, s_{n}, s_{n}^{\prime}, t\right)$ with $s_{n}^{\prime}=\frac{1}{2}$ is relative superior orbit.

Now we define Mandelbrot sets with respect to Ishikawa iterates is named as Relative Superior Mandelbrot set by Negi and Chauhan [19]

\section{Definition 2.4 [15]}

Relative Superior Mandelbrot set $R S M$ for the function of the form $Q_{c}(Z)=z^{n}+c$, where $n=1,2,3, \ldots$ is defined as the collection of $c \in C$ for which the orbit of 0 is bounded

$R S M=\left\{c \in C: Q^{k}{ }_{c}(0): k=0,1,2, \ldots\right\}$ is bounded.

In functional dynamics, we have existence of two different types of points. Points that leave the interval after a finite number are in stable set of infinity. Points that never leave the interval after any number of iterations have bounded orbits. So, an orbit is bounded if there exists a positive real number.

The collection of points that are bounded, i.e. there exists $M$, such that $\left|Q^{n}(z)\right| \leq M$, for all $n$, is called as a prisoner set while the collection of points that are in the stable set of infinity is called the escape set. Hence, the boundary of the prisoner set is simultaneously the boundary of escape set and that is Julia set for $Q$.

\section{Definition 2.5 [3]}

The set of points $\boldsymbol{K}$ whose orbits are bounded under iteration function $Q_{c}(z)$ is called Julia set.

The set of points $S \boldsymbol{K}$ whose orbits are bounded under relative superior iteration of the function $Q(z)$ is called Relative Superior Julia sets. Relative Superior Julia set of $Q$ is boundary of Julia set $R S K$.

\section{GENERATION PROCESS $[5,6]$}

The fractals have been generated by iterative formula: $z_{n+1} \leftarrow f\left(z_{n}\right)$ where $z_{0}=$ is initial value of $z$, and $z_{i}=$ is the value of the complex quantity $z$. The Mandelbrot's self-squared function for generating fractals is $f(z)=z^{2}+c$, where $z$ and $c$ are both complex quantities. We use of the transformation function $z \rightarrow z^{n}+c, n \geq 2$ and $z \rightarrow\left(z^{n}+c\right)^{-1}$ for generating fractal images with respect to Ishikawa iterates, where $z$ and $c$ are the complex quantities and $n$ is a real number. And these fractal images are constructed as a two-dimensional array of pixels. The each pixel is represented by a pair of $(x, y)$ co-ordinates. The complex quantities $z$ and $c$ can be represented as: $z=z_{x}+i z_{y}$ and $c=c_{x}+i c$ Where $i=\sqrt{(-1)}$ and $z_{x}, c_{x}$ are the real parts and $z_{y} \& c_{y}$ are the imaginary parts of $z$ and $c$, respectively. The pixel coordinates $(x, y)$ may be associated with $\left(c_{x}, c_{y}\right)$ or $\left(z_{x}, z_{y}\right)$ which is based on this concept, the fractal images can be classified as follows:

a) $z \quad$-plane fractals, wherein $(x, y)$ is a function of $\left(z_{x}, z_{y}\right)$.

b) $\quad c$-plane fractals, wherein $(x, y)$ is a function of $\left(c_{x}, c_{y}\right)$ In the literature, the fractals for $n=2$ in $z$ plane are termed as the Mandelbrot set while the fractals for $n=2$ in $c$ plane are known as Julia sets [10].

\subsection{Generating the Fractals}

Fractals have been generated from $z \rightarrow z^{n}+c, n \geq 2$ and $z \rightarrow\left(z^{n}+c\right)^{-1}, n \geq 2$ using escape-time techniques, for example by Gujar etal. [5, 6] and Glynn [7]. In this paper we have used escape time criteria of Relative Superior Ishikawa iterates for both of these functions.

\section{Escape Creation [3, 15]}

We obtain a general escape criterion for polynomials of the form $G_{c}(z)=z^{n}+c$

\section{Theorem}

For general functions

$G_{c}(z)=z^{n}+c, n=1,2,3, \ldots$ where

$0<s \leq, 0<s^{\prime}<1$ and $c$ is the complex plane. Define

$z_{1}=(1-s) z+s G_{c}(z)$

$$
z_{n}=(1-s) z_{n-1}+s G_{c}\left(z_{n-1}\right)
$$

The general escape criterion is

$$
\max \left\{|c|,(2 / s)^{1 / n+1},\left(2 / s^{\prime}\right)^{1 / n+1}\right\} \text {. }
$$

\section{Escape Criterion for Quadratics [17]}

Suppose that $|z|>\max \left\{|c|, \frac{2}{s}, \frac{2}{s^{\prime}}\right\}$ then

$|z|>(1+\lambda)^{n}|z|$ 
And $\left|z_{n}\right| \rightarrow \infty$ as $n \rightarrow \infty$.So, $|z| \geq|c|$ and $|z|>2 / s$ as well as $|z|>2 / s^{\prime}$ shows the escape criteria for quadratics [17].

\section{Escape Criterion for Cubic's ${ }^{\text {[17] }}$}

suppose

$|z|>\left\{\max |b|,\left(|a|+\frac{2}{s}\right)^{\frac{1}{2}},\left(|a|+\frac{2}{s^{\prime}}\right)^{\frac{1}{2}}\right\}$ then

$\left|z_{n}\right| \rightarrow \infty$ as $n \rightarrow \infty$. This gives an escape criterion for cubic polynomials [17].

\section{General Escape Criterion [13, 15]}

Let

us consider

$|z|>\left\{\max |b|,\left(|a|+\frac{2}{s}\right)^{\frac{1}{n}},\left(|a|+\frac{2}{s^{\prime}}\right)^{\frac{1}{n}}\right\}$ then

$\left|z_{n}\right| \rightarrow \infty$ as $n \rightarrow \infty$ is the escape criterion. That the

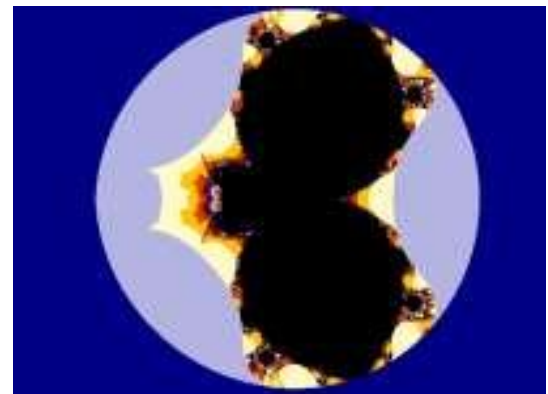

Fig.1: Relative Superior Mandelbrot Set for $\mathrm{s}=\mathbf{0 . 8}$ $s^{\prime}=0.3, n=3.6$

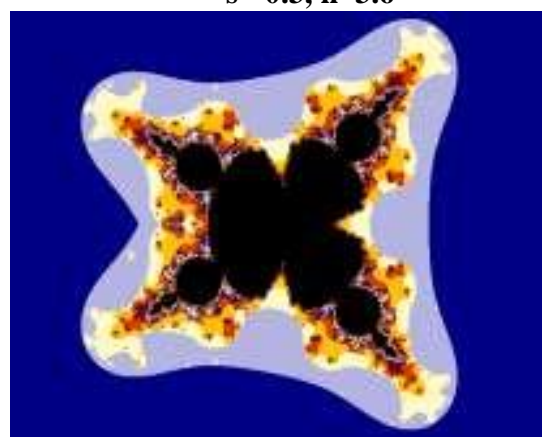

Fig.3: Relative Superior Mandelbrot Set for $\mathrm{s}=0.5$, $s^{\prime}=0.4, n=4.2$ initial value $z_{0}$ should be infinity and the infinity is the critical point of $z \rightarrow\left(z^{n}+c\right)^{-1}$. However instead of starting with $z_{0}=$ infinity, it is simpler to start with $z_{1}=c$, which yields the same result. A critical point of $z \rightarrow F(z)+c$ is a point where $F^{\prime}(z)=0$. The role of critical points is explained in [1].

In the complex dynamics of Ishikawa iterates for non Integer values Negi and Chauhan[17] explore the dynamics of the complex function for non integer values, using the Ishikawa iterates and mathematically analyzed the visual characteristics of the fractal images in the complex $z$ and $c$ planes respectively using the self-squared function $z \rightarrow z^{2}+c$ and transformation of the functions $z \rightarrow z^{2}+c, n \geq 2$ and $z \rightarrow\left(z^{2}+c\right)^{-1}, n \geq 2$ for the cubic and quadratics function, then analyzed the $z$ plane fractal images for the first function and $c$ plane fractals for second function and generated the iterations of these function. There we see the non integer values changes self-similar in the satellite pattern, and the changes occur in the visual characteristics of images from integer value to non integer value.

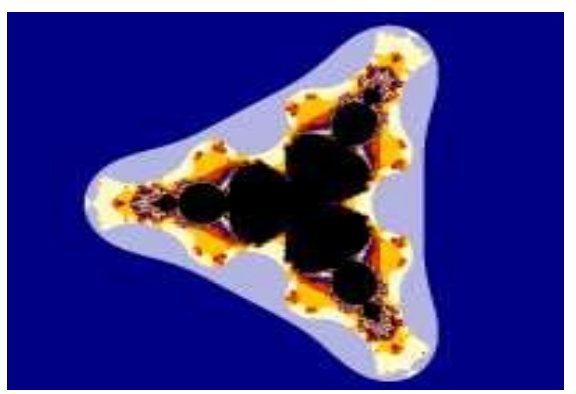

Fig.2: Relative Superior Mandelbrot Set for $s=0.5$ $\mathrm{s}^{\prime}=0.4, \mathrm{n}=4$

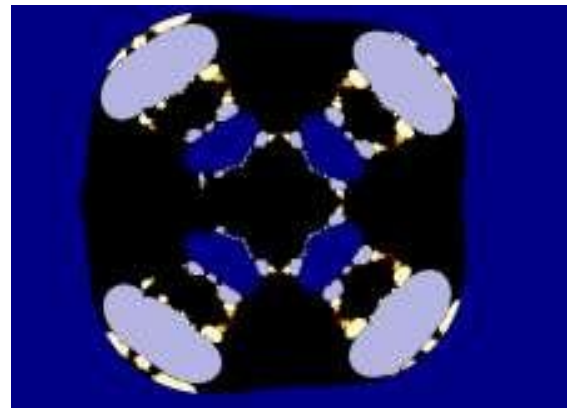

Fig.4: Relative Superior Julia Set for $s=0.5, s^{\prime}=0.4, n=3.2$, $c=0.03488180321-0.01680604855 i$ 


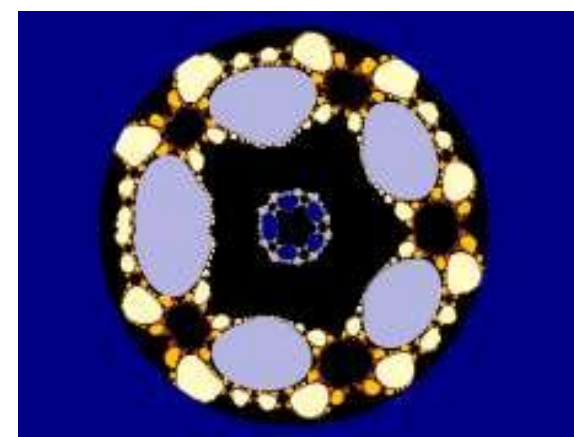

Fig.5:Relative Superior Julia Set $\mathrm{s}=0.8, \mathrm{~s}^{\prime}=0.2$, $\mathrm{n}=4.2, \mathrm{c}=0.04015470798+0.03592299963 \mathrm{i}$

In the Inverse Complex Function Dynamics of Ishikawa Iterates Negi and Chauhan [18] explore the dynamics of the inverse complex function, using the Ishikawa iterates, and mathematically analyzed the visual characteristics of the fractal images in the complex $z$ planes. They have used escape-time methods to produce images of fractals based on the complex mapping $z \rightarrow\left(z^{n}+c\right)^{-1}$ and transformation of the function $z \rightarrow\left(z^{n}+c\right)^{-1}$ for $n \geq 2$ for the cubic er wonder is that they gives satellite like structures which gives a fascinating results in the central planet with satellite like structures.

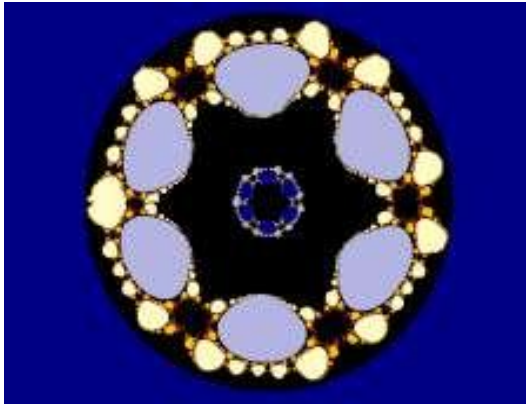

Fig.6: Relative Superior Julia Set $s=0.8, s^{\prime}=0.2$, $\mathrm{n}=4.8, \mathrm{c}=-\mathbf{0 . 0 1 7 8 4 7 2 4 5 7 7 + 0 . 0 3 0 6 5 0 0 9 4 7 4 \mathrm { i }}$

and quadratics functions. Further, they have analyzed the $z$ plane fractal images generated from the iterations of this function using Ishikawa iteration procedure and noticed the drastic changes that occur in the visual characteristics of the images. Relative Superior Mandelbrot of inverse function showed lace like structures with small circles and their relative superior Mandelbrot and relative superior Julia sets of inverse function showed their reflection symmetry there is anoth

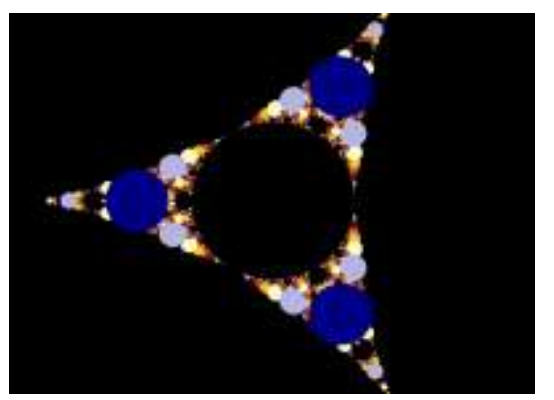

Fig.7: Relative Superior Mandelbrot Set for $s=s^{\prime}=1$

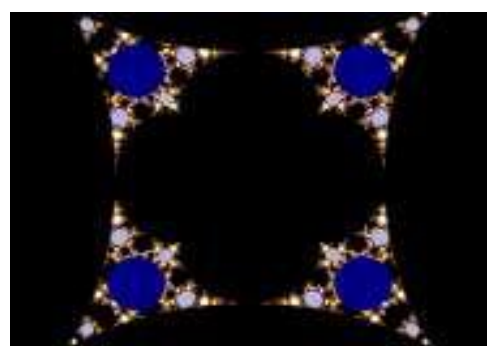

Fig.9: Relative Superior Mandelbrot Set for $s=s^{\prime}=1$

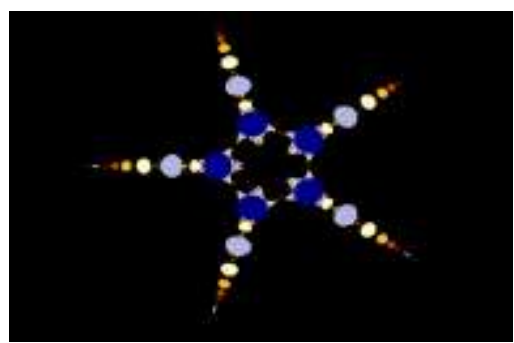

Fig.11: Relative Superior Mandelbrot Set for $s=0.5, s^{\prime}=0.3$

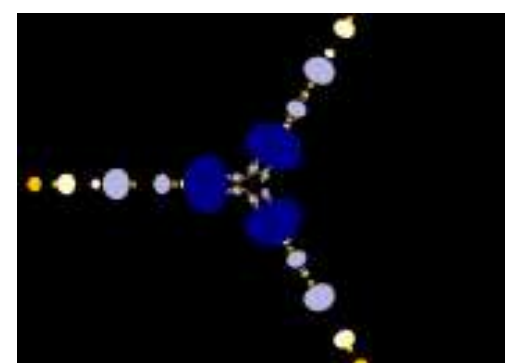

Fig.8: Relative Superior Mandelbrot Set for $s=0.5, s^{\prime}=0.1$

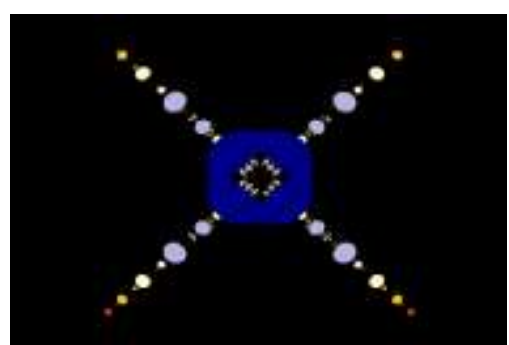

Fig.10: Relative Superior Mandelbrot Set $s=0.5, s^{\prime}=0.1$

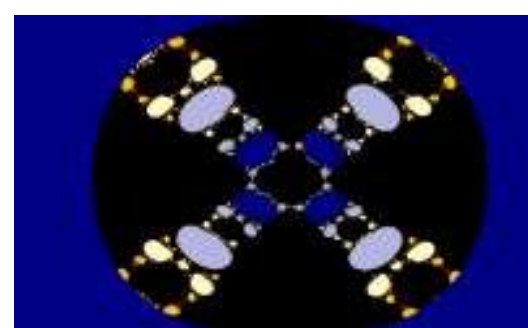

Fig.12: Relative Superior Julia Set for $s=0.5, s^{\prime}=0.3$, c $=-$ $0.02051433067+0.012696182776 i$ 
In the paper New Julia Sets of Ishikawa iteration Negi and Chauhan [20] an idea of orbit traps in a complex plane and explores their relative fractals images actually an orbit trap is a bounded area into which all orbiting points are fall. They uses different types of orbit traps for Ishikawa iteration procedure we know that the Julia sets of $z_{n-1}=a z_{n}^{2}+c$ are connected and bounded for $a$ and $c$, for the cubic and quadratics function, for a quadratics they

\begin{tabular}{|l|l|l|l|}
\hline $\begin{array}{c}\text { Number } \\
\text { of } \\
\text { iteration i }\end{array}$ & $|\mathbf{F}(\mathbf{z})|$ & $\begin{array}{l}\text { Number of } \\
\text { iteration } \mathbf{i}\end{array}$ & $|\mathbf{F}(\mathbf{z})|$ \\
\hline 81 & 0.9200 & 96 & 0.9199 \\
\hline 82 & 0.9196 & 97 & 0.9200 \\
\hline 83 & 0.9197 & 98 & 0.9200 \\
\hline 84 & 0.9201 & 99 & 0.9198 \\
\hline 85 & 0.9201 & 100 & 0.9198 \\
\hline 86 & 0.9198 & 101 & 0.9200 \\
\hline 87 & 0.9197 & 102 & 0.9200 \\
\hline 88 & 0.9200 & 103 & 0.9199 \\
\hline 89 & 0.9201 & 104 & 0.9198 \\
\hline 90 & 0.9199 & 105 & 0.9199 \\
\hline 91 & 0.9197 & 106 & 0.9200 \\
\hline 92 & 0.9199 & 107 & 0.9199 \\
\hline 93 & 0.9200 & 108 & 0.9199 \\
\hline 94 & 0.9199 & 109 & 0.9199 \\
\hline 95 & 0.9198 & 110 & 0.9199 \\
\hline
\end{tabular}

Table-1:Orbit of $\mathrm{F}(\mathrm{z})$ for $(\mathrm{z0}=0.134+0.128 \mathrm{i})$ at $\mathrm{s}=0.3$ and $\mathrm{S}^{\prime}=\mathbf{0 . 4} \mathrm{s}^{\prime}=\mathbf{0 . 4}$

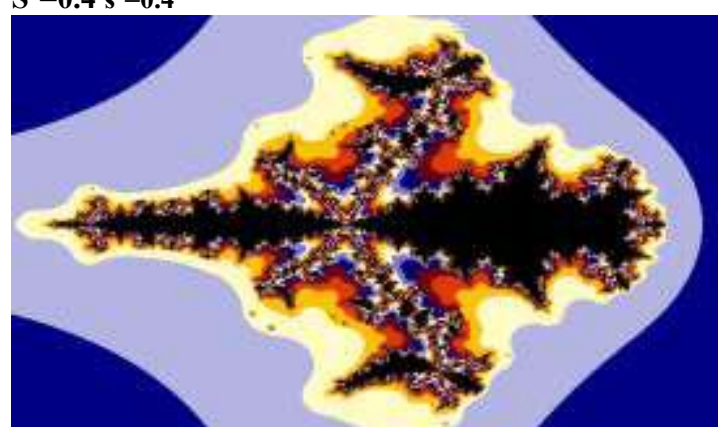

Fig20: Relative Superior Julia Set for $\mathrm{s}=0.3, \mathrm{~s}^{\prime}=0.4, \mathrm{c}=$ $3.6+0.0 \mathrm{i}$

\section{CONCLUSION}

Ishikawa Iterates are an example of a two-step feedback machine, and have proved their usefulness in the study of fractals. In all these four paper we explore the work done by the Negi and Chauhan using the Ishikawa iterates analyzed the visual characteristics of the fractal images in the complex planes respectively as well as they also define the Relative Superior Mandelbrot set and use the concept of orbit traps to describe the fractals more fascinating way. In their study we observe various remarkable fractals and their fixed points given above. Their work opens a scope of research in the study of new Fractal Model using the two step feedback machine

\section{ACKNOWLEDGMENTS}

Our thanks to Dr. A.K. Swami, Principal, G.B.Pant Engineering College, Ghurdauri, for providing necessary infrastructure for the research work. We would also like to thank Mr. Yashwant Chauhan, Assistant Professor, Department of Computer Science and Engineering, G.B.Pant Engineering College, Ghurdauri , Mrs. Rajshri takes function $Q_{c}=z^{2}+c$, for cubic takes $Q_{a, b}(z)=z^{3}+a z+b$ with respect to the Ishikawa iteration procedure and calculated the iterations values. When they take a numerical analysis of fixed-point iteration of computing fixed points of iterated function is,

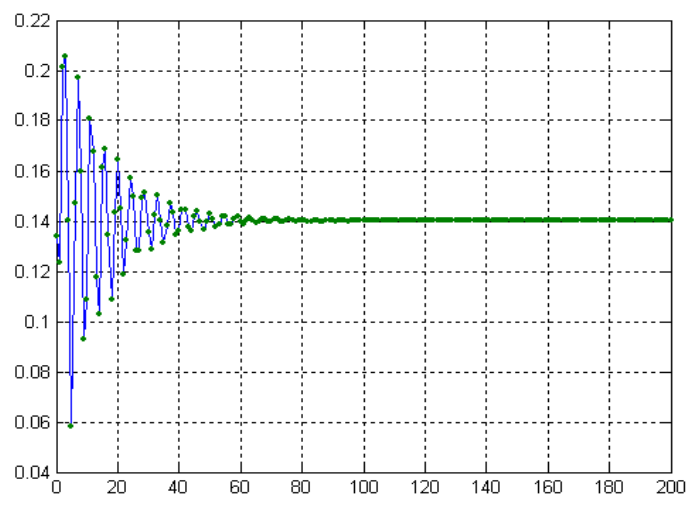

Fig19: Orbit of $\mathrm{F}(\mathrm{z})$ for $(\mathrm{z0}=0.134+0.128 \mathrm{i})$ at $\mathrm{s}=0.3$ and

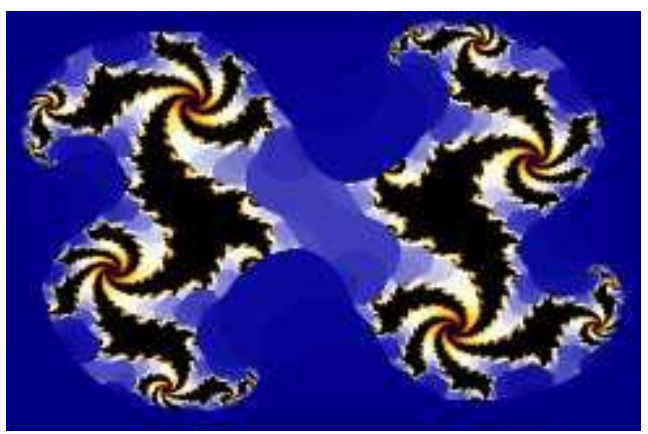

Fig21: Relative Superior Julia Set for $s=1, s^{\prime}=0.3$, $\mathrm{c}=\mathbf{0 . 4 3 0}+\mathbf{0 . 1 8} i$

Rana Chauhan, Assistant Professor, Department of Applied science and humanities, G.B.Pant Engineering College, Ghurdauri for their unconditional and valuable technical support in writing this paper.

\section{REFERENCES}

[1] B. Branner, "The Mandelbrot Set", Proceedings of Symposia in Applied Mathematics39 (1989), 75-105.

[2] P. Blanchard, "Complex Analytic Dynamics on the Riemann Sphere", Bulletin of the American Mathematical Society 11, 1 (1984), 85-141.

[3] P. Blanchard, "Complex Analytic Dynamics on the Riemann Sphere", Bulletin of the American Mathematical Society 11, 1 (1984), 85-141.

[4] S. Dhurandar, V. C. Bhavsar and U. G. Gujar, "Analysis of z-plane fractal images from $z \leftarrow z^{\alpha}+c$ for $\alpha<0$ ", Computers and Graphics 17, 1 (1993), 89-94. 
[5] U. G. Gujar and V. C. Bhavsar, "Fractals from $z \leftarrow z^{\alpha}+c$ in the Complex c-Plane", Computers and Graphics 15, 3 (1991), 441-449.

[6] U. G. Gujar, V. C. Bhavsar and N. Vangala, "Fractals from $z \leftarrow z^{\alpha}+c$ in the Complex z-Plane", Computers and Graphics 16, 1 (1992), 45-49.

[7] E. F. Glynn, "The Evolution of the Gingerbread Mann", Computers and Graphics 15,4 (1991), 579-582.

[8] S. Ishikawa, "Fixed points by a new iteration method", Proc. Amer. Math. Soc.44 (1974), 147-150.

[9] G. Julia, "Sur 1' iteration des functions rationnelles", J Math Pure Appli. 8 (1918), 737-747.

[10] K. W. Shirriff, "An investigation of fractals generated by $z \rightarrow z^{-n}+c$ ", Computers and Graphics 13, 4 (1993), 603-607.

[11] B. B. Mandelbrot, "The Fractal Geometry of Nature", W. H. Freeman, New York, 1983.

[12] H. Peitgen and P. H. Richter, "The Beauty of Fractals", Springer-Verlag, Berlin,1986.

[13] Peitgen H, Jürgens H, Saupe D. Chaos and fractals: new frontiers of science. New York: Springer-Verlag; 2004.

[14] C. Pickover, "Computers, Pattern, Chaos, and Beauty", St.
Martin's Press, NewYork, 1990.

[15] R. Rana, Y. S. Chauhan and A. Negi, Non Linear dynamics of Ishikawa Iteration, In Press, Int. Journal of Computer Application (Oct. 2010 Edition).

[16] S. T. Welstead and T. L. Cromer, "Coloring Periodicities of Two-dimensional Mappings",

Computers and Graphics 13, 4 (1989), 539-543.

[17] R. Rana, Y. S. Chauhan and A. Negi, Complex dynamics of Ishikawa iterates for non Integer values, In Press, Int. Journal of Computer Application (Nov. 2010 Edition).

[18] R. Rana, Y. S. Chauhan and A. Negi, Inverse Complex Function Dynamics of Ishikawa Iterates, In Press, Int. Journal of Computer Application (Nov. 2010 Edition).

[19] R. Rana, Y. S. Chauhan and A. Negi, Non Linear Dynamics of Ishikawa iteration, In Press, Int. Journal of Computer Application (Nov. 2010 Edition).

[20] R. Rana, Y. S. Chauhan and A. Negi, New Julia Sets of Ishikawa iteration, In Press, Int. Journal of Computer Application (Oct. 2010 Edition). 